\title{
ASPECTOS TOXICOLÓGICOS DE LA EXPOSICIÓN AL ÓXIDO DE ETILENO
}

\author{
Andrés Prat Marín*
} Pedro Sanz Gallén*

PRAT-MARIN, A. \& SANZ-GALLEN, P. Aspectos toxicológicos de la exposición al óxido de etileno. Rev. Saúde públ., S. Paulo, 21:523-8, 1987.

RESUMEN: En este trabajo se revisan los principales riesgos de la utilización del óxido de etileno como agente esterilizante del material médico-quirúrgico. Resaltando los efectos tóxicos, mutagénicos, teratogénicos y cancerígenos como los más importantes, enfatizando en la necesidad de adoptar medidas de prevención para controlar dichos riesgos.

DESCRIPTORES: Oxido de etileno, toxicidad. Esterilización. Riesgo.

\section{INTRODUCCIÓN}

E1 empleo de diferentes substancias con una finalidad industrial o sanitaria, puede comportar riesgos de diversa naturaleza que interesa conocer adecuadamente. El óxido de etileno es un gas que, a temperatura y presión normales, tiene una densidad superior a la del aire $\mathrm{y}$ es soluble en agua. Por su estructura química (Figura) pertenece al grupo de los

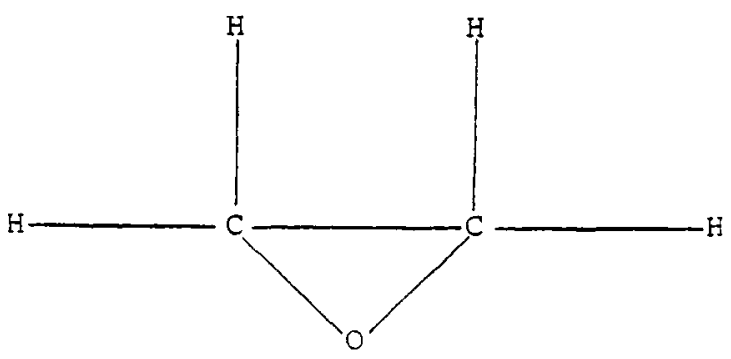

Fig. - Estructura Química del Oxido de Etileno

epoxy compuestos. Este oxileno o enlace epoxy es extremadamente reactivo con todo tipo de material que entra en contacto con él. El óxido de etileno actua combinándose con los grupos carboxilo, amino, sulfhidrilo e hidroxilo de las proteínas bacterianas. Para evitar hipotéticas inflamaciones se utiliza generalmente mezclado con gases inertes; cuando se le comparan sus propiedades esterilizantes con las de otros agentes químicos (óxido de propileno, formalina, glicoles, etilenimina, halóge- nos, glutaraldehido, etc.), es el mas efectivo y el menos peligroso de todos ellos. Actualmente es una de las 25 substancias químicas mas utilizadas mundialmente ${ }^{37,43}$.

Sin embargo, en los últimos años se ha cuestionado su uso, como resultado de diversas investigaciones, en las que, se le imputan unos posibles efectos mutagénicos, teratogénicos y cancerígenos $\mathrm{s}^{13,20,43}$.

Debido a su amplia utilización en el medio hospitalario como agente esterilizante del material médico-quirúrgico (Tabla 1), efectuamos una revisión y puesta al día de esta incipiente temática.

\section{TABLA 1}

Composición del material esterilizable por óxido de etileno.

Plástico: Catéteres, sondas, jeringuillas, tubos, nebulizadores, etc.

Vídrio: Termômetros, pipetas, probetas, matraces, etc.

Metal: Agujas, bisturis, tijeras, otoscopios, oftalmoscopios, citoscopios, proctoscopios, microscopios, forceps, etc.

Tela: Mantas, almohadas, colchones, etc.

Madera: Depresores, férulas, torundas, etc.

Papel: Libros, periódicos, paquetes, etc.

Cuero: Zapatos, maletas, etc.

Goma: Guantes, tubos, etc.

* Departamento de Salud Pública y Legislación Sanitaria da Universidad de Barcelona - Avda. Diagonal, s/n. - 08028 - Barcelona, España. 


\section{TOXICOCINÉTICA}

Las principales vías de entrada del óxido de etileno en el organismo son a través del contacto cutáneo y de la inhalación; una vez que ha penetrado se redistribuye por todos sus compartimientos.

$\mathrm{Su}$ vida media es corta eliminándose un $78 \%$ durante las primeras 48 horas, según diversos estudios experimentales ${ }^{10}$.

Al reaccionar con los grupos glutation, se forman principalmente dos metabolitos que serán eliminados a través de la vía renal: la $\mathrm{S}$-(2-hidroxietil)cisteína y la N-acetil-S-(2-hidroxietil)cisteina ${ }^{11}$.

Recientemente, Wolfs e col ${ }^{49}$ han publicado un estudio realizado sobre 18 trabajadores expuestos laboralmente al óxido de etileno en una central de esterilización de instrumental médico-quirúrgico. Al encontrar respecto a un grupo control no expuesto, un aumento significativo de etilenglicol en sangre, estos autores presuponen que una fracción del óxido de etileno absorvido sufre una hidratación que originaría dicho aumento.

\section{EFECTOS TOXICOS}

La acción del óxido de etileno sobre el organismo puede ocasionar una serie de efectos tóxicos que los clasificaremos en diversos apartados.

\section{Intoxicación Aguda}

Dependiendo de la intensidad de la exposición, la inhalación de óxido de etileno puede provocar las siguientes manifestaciones clínicas: irritación de las mucosas conjuntival, nasal y bucal, alteraciones respiratorias (dísnea, cianosis, edema pulmonar), alteraciones gastrointestinales (nauseas, vómitos) y alteraciones neurológicas (cefaleas, somnolencia, debilidad muscular, convulsiones e incoordinación motora). La sintomatología suele debutar después de un periodo asintomático que puede durar entre una y varias horas ${ }^{11,42}$. Los accidentes laborales suelen ser la causa de estas intoxicaciones de carácter agudo. En 1986 se han publicado en España tres casos de intoxicación aguda de óxido de etileno en medio hospitalario, que cursaron con una total recuperación pocos días después ${ }^{23}$. Cuando se inhalan durante varios minutos concentraciones que sobrepasan los $500-700 \mathrm{ppm}$, aparece la sintomatología de mayor gravedad (edema pulmonar y convulsiones) ${ }^{13}$. La congestión ce- rebral, pulmonar y meníngea son hallazgos anatomo-patológicos inespecíficos descritos en los casos mortales ${ }^{14}$.

\section{Intoxicación Crónica}

Las manifestaciones clínicas que se presentan en este tipo de intoxicación son neurológicas $^{13,43}$. Se han descrito casos de encefalopatía y de neuropatías periféricas ${ }^{14}$ así como alteraciones neurovegetativas. Estas manifestaciones neurológicas tienen un caráter progresivamente reversible al cesar la exposición al gas $^{13,14}$. Conjuntamente a las alteraciones descritas, los riesgos de la exposición a largo plazo al óxido de etileno, incluso dentro de los niveles ambientales permitidos, se centran actualmente en sus posibles efectos mutágenos, teratógenos y cancerígenos que serán expuestos en el siguiente apartado.

\section{Efectos Mutagénicos, Cancerígenos, Teratogénicos y de la Función Reproductora}

Si una molécula puede alquilar el DNA y engendrar mutaciones genéticas y cromosómicas en diferentes sistemas "in vitro" $e$ "in vivo", se considera que posee un riesgo mutágeno y cancerígeno ${ }^{13}$. Existen varios estudios que revelan el efecto mutágeno del óxido de etileno sobre microorganismos ${ }^{37}$, la mosca drosophila melanogaster ${ }^{34}$, vegetales ${ }^{33}$ y diversos animales mamíferos". El óxido de etileno "in vitro" produce aberraciones cromosómicas y un aumento de las cromatides hermanas en las células humanas ${ }^{38}$.

Para investigar los efectos que produce el óxido de etileno en los individuos laboralmente expuestos, en los últimos años se han realizado estudios citogenéticos en linfocitos

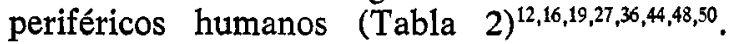
En la casi totalidad de estos trabajos se han podido corroborar las alteraciones encontradas previamente "in vitro".

Si bien a nivel experimental diversas publicaciones atribuyen algunos tipos de neoplasia al óxido de etileno ${ }^{26,28}$, los estudios epidemiológicos observacionales realizados en poblaciones humanas proporcionan resultados muy contradictorios. Así Hogsted y $\operatorname{col}^{18,20}$ han encontrado en diferentes estudios efectuados sobre colectivos laboralmente expuestos, una incidencia superior a la esperada de cáncer gástrico y de leucemias ${ }^{18,20}$. A raiz de su última publicación ${ }^{20}$, Hogsted y col. se han visto obligados a replicar y defender la metodología empleada en sus investigaciones ${ }^{21}$, al haber si- 
TABLA 2

Estudios de mutagenicidad para el óxido de etileno

\begin{tabular}{|c|c|c|c|}
\hline Autores & Casos & Efecto linfocitario & T.L.V. \\
\hline $\begin{array}{l}\text { Garry e col. }{ }^{13} \\
(1979)\end{array}$ & 16 & Aumento cromátides & $36 \mathrm{ppm}$ \\
\hline $\begin{array}{l}\text { Pero e col. }{ }^{37} \\
(1981)\end{array}$ & 17 & $\begin{array}{l}\text { Aberraciones } \\
\text { cromosómicas }\end{array}$ & $0,5-1,2 \mathrm{ppm}$ \\
\hline $\begin{array}{l}\text { Thiess e col. } 49 \\
\text { (1981) }\end{array}$ & 43 & $\begin{array}{l}\text { Aberraciones } \\
\text { cromosómicas }\end{array}$ & $3 \mathrm{ppm}$ \\
\hline $\begin{array}{l}\text { Yager e col.51 } \\
(1983)\end{array}$ & 5 & Aumento cromátides & $0,92 \mathrm{ppm}$ \\
\hline $\begin{array}{l}\text { Hogsted e col. }{ }^{20} \\
(1983)\end{array}$ & 28 & $\begin{array}{l}\text { Aberraciones } \\
\text { cromosómicas }\end{array}$ & 1 ppm \\
\hline $\begin{array}{l}\text { Laurent e col.28 } \\
(1983)\end{array}$ & 25 & Aumento cromátides & - \\
\hline $\begin{array}{l}\text { Sarto e col.45 } \\
(1984)\end{array}$ & 22 & $\begin{array}{l}\text { Aberraciones } \\
\text { cromosómicas }\end{array}$ & $0,35 \mathrm{ppm}$ \\
\hline$\underset{(1984)}{\text { Hansen }}$ e col. ${ }^{17}$ & 14 & Negativo & $5 \mathrm{ppm}$ \\
\hline
\end{tabular}

do ésta cuestionada por representantes de la Texaco Inc. ${ }^{8}$ Según éstos no se había tenido en cuenta la posible influencia de otras substancias que podían estar presentes.

Por otra parte, dos estudios realizados, uno de ellos por Thiess y col. ${ }^{47} \mathrm{y}$ el otro por Morgan y col. ${ }^{29}$ en trabajadores expuestos al óxido de etileno, no han encontrado una mayor incidencia de neoplasias.

Simultaneamente, algunas agencias internacionales se han manifestado respecto a la gran controversia existente sobre la cancerogenicidad de este gas. Tanto la $\mathrm{NIOSH}^{35}$, como la ECETOC $^{11}$ y la IARC ${ }^{22}$ manifiestan, de acuerdo a los hallazgos experimentales, el posible potencial cancerígeno del óxido de etileno, y expresan la necesidad de que se efectuen un mayor número de estudios epidemiológicos con el fin de establecer unas conclusiones más claras.

Experimentalmente se ha comprobado que el óxido de etileno produce alteraciones tanto embrionarias como fetales ${ }^{24,46}$, y disminuye la función reproductora ${ }^{45}$. Aunque la información a nivel humano es muy escasa, hay que destacar el estudio realizado por Hemminki y col. ${ }^{17}$ en Finlandia. En este trabajo se han encontrado diferencias significativas en la incidencia de abortos espontáneos en trabajadoras expuestas laboralmente al óxido de etileno durante el periodo de gestación, con respecto a la incidencia de abortos espontáneos esperados según los estudios epidemiológicos realizados en dicho pais.

\section{Reacciones Alérgicas}

El óxido de etileno es un irritante de piel y mucosas ${ }^{15}$. Como la mayoría de las substancias que pertenecen al grupo epoxy, su capacidad sensibilizante es considerable ${ }^{13,15,43}$, pudiendo producir dermatitis de tipo alérgico que se confirman con la positividad de los tests epicutáneos ${ }^{1}$.

Se han observado casos de anafilaxia en pacientes tratados con material esterilizado con esta substancia. Poothuill y $\mathrm{col}^{40}$ han descrito una reacción de hipersensibilidad tipo I en un enfermo hemodializado, al que se le había conectado un shunt arterio-venoso esterilizado previamente con óxido de etileno. La positividad del test epitutáneo para una solución de óxido de etileno/albúmina sérica humana, la liberación de histamina a partir de leucocitos incubados en presencia del mismo complejo, y la detección de anticuerpos circulantes vis-a-vis de óxido de etileno', han confirmado estas reacciones de carácter alérgico. Varios trabajos publicados más recientemente indican la alta incidencia de anticuerpos y la presencia de reacciones anafilácticas y de hipersensibilidad, en pacientes hemodializados con material esterilizado con este gas $^{5,41}$. 


\section{ASPECTOS PREVENTIVOS}

La prevención actual de los riesgos toxicológicos del óxido de etileno se basa principalmente en el control de las concentracciones del gas. Este principio debe considerarse desde una perspectiva ocupacional de las personas expuestas, y desde una perspectiva clínica de los pacientes tratados con materiales esterilizados con dicho gas.

La mayor parte de los paises industrializados han legislado las concentraciones máximas permisibles de óxido de etileno en el medio ambiente. La ACGIH (American Conference of Governmental Industrial Hygienists) de USA, que había reducido de 50 a $10 \mathrm{ppm}$ el TLV (valor medio de concentración durante una jornada laboral de 40 horas semanales), propone en 1984 la cifra de 1 $\mathrm{ppm}^{2}$. En la URSS está limitado a $0,5 \mathrm{ppm}^{6}$. En Italia, que en 1981 estaba regulado en 50 ppm*, pasó en 1983 a 3 ppm $^{32}$. Francia está legislado en $5 \mathrm{ppm}^{25}$. En España, está contemplado de un modo muy genérico en el Decreto regulador de actividades molestas, insalubles, nocivas y peligrosas de 1961, fijando el tope máximo en $50 \mathrm{ppm}^{7}$.

Debido a las complicaciones técnicas de laboratorio y a la escasez de estudios concluyentes, no hay todavía ningún test biológico que se pueda utilizar en los individuos expuestos al óxido de etileno $0^{43}$.
Algunos paises tienen legislada la cantidad tolerable de óxido de etileno residual que puede estar presente en el material esterilizado. Francia, por ejemplo, lo tiene establecido en $2 \mathrm{ppm}^{30}$. Para ello, una vez finalizado el ciclo de esterilización, el material debe seguir un proceso de aireación previo a su utilización sanitaria ${ }^{31}$. Según el tipo de cámara y las condiciones de la sala de esterilización, el tiempo de aireación puede variar entre una hora y varios dias dependiendo fundamentalmente el tipo de material que se esterilize ${ }^{39}$.

Por todo ello, resultan imprescindibles unas normas básicas de utilización del óxido de etileno como agente esterilizante. El empleo de otros sistemas alternativos de esterilización si la naturaleza del material lo permite, una persona responsable que supervise la totalidad del proceso, un servicio de revisión y mantenimiento periódicos de los aparatos y la realización de controles ambientales y residuales, son las principales recomendaciones en este tipo de esterilización ${ }^{4}$. Se bién otros métodos de esterilización van incorporándose al mercado industrial y sanitario (radiaciones gam$\mathrm{ma}$ ), es previsible que el óxido de etileno siga siendo el agente esterilizante más utilizado durante muchos años. De ahí la necesidad de definir criterios, potenciar investagaciones y controlar actividades relacionadas con dicho gas para prevenir al máximo dos riesgos que comporta su utilización en el medio industrial y sanitario.

PRAT-MARÍN, A. \& SANZ-GALLÉN, P. Aspectos toxicológicos da exposição ao óxido de etileno. Rev. Saúde públ., S. Paulo, 21:523-8, 1987.

RESUMO: Neste trabalho são revisados os principais riscos de utilização do óxido de etileno como agente esterilizante do material médico-cirúrgico. Ressaltam-se efeitos tóxicos, mutagênicos, teratogênicos e cancerígenos como sendo os mais importantes, enfatizando a necessidade de adotar medidas de prevenção para controlar os referidos riscos.

UNITERMOS: óxido de etileno, toxicidade. Esterilização. Risco.

PRAT-MARÍN, A. \& SANZ-GALLÉN, P. [Toxicological aspects of the exposure to ethylene oxide]. Rev. Saúde públ., S. Paulo, 21:523-8, 1987.

ABSTRACT: Some important aspects of the use of ethylene oxide as a sterilizing agent for medical instruments are reviewed in this paper. Toxic, mutagenic, teratogenic and carcinogenic effects are the main risks described and emphasis it given to preventive mesures.

UNITERMS: Ethylene oxide, toxicity. Esterilization. Risk.

* ASCHIMICI: Promemoria ossido di etilene (Circolare) Milano 29 ottobre 1981. 
REFERÊNCIAS BIBLIOGRÁFICAS

1. ALOMAR, A. et al. Ethylene oxide dermatitis. Contact Dermatitis, 7:205-7, 1981.

2. AMERICAN CONFERENCE OF GOVERNMENTAL INDUSTRIAL HYGIENISTS. Documentation of the TLV's for substances in workroom air. $3^{\text {rd }}$ ed. Cincinnati, Ohio. 1971.

3. APPELGREN, L. E. et al. Testing of ethylene oxide for mutagenicity using the micronucleus test in mice and rats. Acta pharmacol. toxicol., 43:69-71, 1978.

4. ASSOCIATION FOR THE ADVANCEMENT OF MEDICAL INSTRUMENTATION. Ethy. lene oxide gas ventilation: recomendations and safe use. Missouri, 1981.

5. BOMMER, J. et al. Anaphylactoid reactions in dialysis patients: role of ethylene oxide. Lancet, 2:1382-4, 1985.

6. CAHIER DE NOTES DOCUMENTAIRES. Paris, (98) 1980. [Note n. ${ }^{\circ} 1231$ - 98-80].

7. DECRETO $2414 / 1961$ sobre Reglamento de actividades molestas, insalubres, nocivas y peliglosas. Bol. ofic. Estado, Madrid, (292) 7 dic. 1961 .

8. DIVINE, D. J. \& AMANOLLAHI, K. S. Ethylene oxide and cancer. J. Amer. med. Ass., 256: $1726-7,1986$.

9. DOLOVICH, J. \& BELL, B. Allergy to products of ethylene oxide gas. J. Allergy clin. Immunol, 62:30-2, 1978.

10. EHRENBERG, L. et al. Evaluation of genetic risks of Alkylating agents. Tissue doses in the mouse from air contaminated with ethylene oxide. Mutat. Res., 24:83-103, 1974.

11. EUROPEAN CHEMICAL INDUSTRY ECOLOGY TOXICOLOGY CENTRE (ECETOC). Toxicity of ethylene oxide and its relevance to man. Brussels, 1982. (Technical report n. ${ }^{\circ}$ 5).

12. GARRY, V. F. et al. Ethylene oxide. Evidence of human chromosomal effects. Environ. Mutagen., 1:375-82, 1979.

13. GENNART, J. et al. La toxicité de l'oxyde d'ethylene. Arch. Mal. prof., 44:269-74, 1983.

14. GROSS, J. A. et al. Ethylene oxide neurotoxicity: report of four cases and review of the literature. Neurology, 29:978-83, 1979.

15. HANIFIN, M. J. Ethylene oxide dermatitis. J. Amer. med. Ass., 217:213, 1971.

16. HANSEN, J. P. et al. Normal sister chromatid exchange levels in hospital sterilisation employes exposed to ethylene oxide. J. occup. Med., 26:29-32, 1984.

17. HEMMINKI, L. et al. Spontaneus abortions in hospital staff engaged in sterilising instruments with chemical agents. Brit. med. J., 285: 1461-3, 1982.
18. HOGSTEDT, C. et al. Leukemia in workers exposed to ethylene oxide. J. Amer. med. Ass., 241:1132-3, 1979.

19. HOGSTEDT, $C$. et al. Chromosome aberrations and micronuclei in bone marrow cells and peripheral bl not lymphocytes in human exposed to ethylene oxide. Hereditas, 98: 105-13, 1983.

20. HOGSTEDT, C. et al. Epidemiologic support for ethylene oxide a cancer causing agent. J. Amer. med. Ass., 255:1575-8, 1986.

21. HOGSTEDT, C. In reply: ethylene oxide and cancer. J. Amer. med. Ass., 256:1727, 1986.

22. INTERNATIONAL AGENCY FOR RESEARCH ON CANCER (IARC). Chemicals industrial processes and industries associated with human cancer. Lyon, 1982. (IARC Monographs Supplement 4).

23. JULIĀ, L. C. et al. Intoxicación aguda por óxido de etileno. Med. clin., Barcelona, 86:350, 1986.

24. LA BORDE, J. M. \& KIMMEL, C. A. The teratogenicity of ethylene oxide administered intravenously to mice. Toxicol. appl. Pharmacol., 56:16-22, 1980.

25. LA FOREST, J. C. et al. Metrologie des ambiances toxiques et valeurs limites de concentration des substances dangereuses dans l'atmosphere des locaux de travail. In: Encyclopedie Medico-chirurgicale. Paris, 1987. Intoxications 160001 A 16, 2-1987, 8P, 1987.

26. LANDRIGAN, P. J. et al. Ethylene oxide: an overview of toxicologic and epidemiologic research. Amer. J. industr. Med., 6:103-15, 1984.

27. LAURENT, C. H. et al. Sister chromatid exchange frequency in workers to high levels of ethylene oxide, in a hospital sterilization service. Int. Arch. occup. environ. Hlth, 54: 33-43, 1984.

28. LYNCH, D. W. et al. Carcinogenic and toxicologic effects of inhaled ethylene oxide and propylene oxide in F 344 rats. Toxicol. appl. Pharmacol., 76:69-84, 1984.

29. MARGAN, R. W. et al. Mortality among ethylene oxide workers J. occup. Med., 23: 767-70, 1981.

30. MAUPETIT, B. et al. Les risques dus a la sterilisation par l'oxide d'ethylene, existent-ils encore? Anesth. Anal. Paris, 35:1214-21, 1978.

31. McGUNNIGLE, R. G. et al. Residual ethylene oxide: levels in medical grade tubing and effects on an "in vitro" biologic system. J. biomed. Mater. Res., 9:273-83, 1975.

32. MINISTERO DELL SANITA. Ossido di etilene: modifica della circolare $n .^{\circ} 47$ dell 1-10-1981 e svolgimento indagine epidemiologica. Roma, 1983. (Circolare n. ${ }^{\circ} 8$ ). 
33. MOUTSCHEN, J. et al. Note on the chromosome breaking activity of ethylene oxide and ethyleneimine. Hereditas, 60:267-9, 1968.

34. NAKAO, Y. \& AUERBACK, C. Test of a possible correlation crosslinking and chromosome breaking abilities to chemical mutagens. Z. Vererbungsl., 92:457-61, 1961.

35. NATIONAL INSTITUTE FOR OCCUPATIONAL SAFETY AND HEALTH (NIOSH). Ethylene oxide (EtO): evidence of carcinogenicity. Current Intelligence Bulletin (35), 1981.

36. PERO, R. W. et. al. In vivo and in vitro ethylene oxide exposure of human lymphocytes assessed by chemical stimulation of unscheduled DNA synthesis. Mutat. Res., 83:271-89, 1981 .

37. PFIFFER, E. \& DUNKELBERG, H. Mutagenicity of ethylene oxide and propylene oxide and the glycols and halahydrins formed from them during the fumigation of foodstuffs. Food Cosmet. Toxicol., 18:115-8, 1980.

38. POIRIER, V. \& PAPADOPOULO, D. Chromosomal aberrations induced by ethylene oxide in a human amniotic cell line in vitro. Mutat. Res., 104:255-60, 1982.

39. PONS, M. et al. Esterilització amb oxid d'etilé, problemes toxicològics i la seva reperdusió a l'àmbit hospitalari. Gas. sanit., 2:24-30. 1983.

40. POOTHUILL, J. et al. Anaphylaxis from the product(s) of ethylene oxide gas. Ann. intern. Med., 82:58-60, 1975.

41. ROCKEL, A. et al. Ethylene oxide hypersensitivity in dialysis patients. Lancet, 1:382-3, 1986.

42. SALINAS, E. et al. Acute ethylene oxide intoxication. Drug Intell. clin. Pharm., 15:384-6, 1981.
43. SARTO, F. Tossicitá dell ossidio di etilene e sua rilevanza per l'uomo. Med. Lav., 75: 254-63, 1984.

44. SARTO, $F$. et al. Cytogenetic damage in workers exposed to ethylene oxide. Mutat. Res., 138:185-95, 1984.

45. SNELLINGS, W. M. et al. Effects on reproduction in fischer 344 rats exposed to ethylene oxide by inhalation for one generation. Toxicol. appl. Pharmacol., 63:382-8, 1982.

46. SNELLINGS. W. M. et al. Teratology study in fischer 344 rats exposed to ethylene oxide by inhalation. Toxicol. appl. Pharmacol., 64: 476-81, 1982.

47. THIESS, A. M. et al. Mortality study on employees also exposed to alkylene oxides and its derivate. In: Internal Symposiun on Prevention of Occupational Cancer, Proceedings. Helsinki, Finland, 1981. p. 249-59.

48. THIESS, A. M. et al. Mutagenicity study of workers exposed to alkylene oxide (ethylene oxide/propylene oxide) and derivates. $J$. occup. Med., 23:343-7, 1981.

49. WOLFS, P. et al. Surveillance des travailleurs exposés à l'oxyde d'éthylene dans une entrepise de distribution de gaz stérilisants et dans des unités de stérilisation de matérial medical. Arch. Mal. prof., 44:321-8, 1983.

50. YAGER, J. W. et al. Exposure to ethylene oxide at work increases sister chromatid exchanges in human peripheral lymphocytes. Science, 219:1221-3, 1983.

Recebido para publicação em: 26/6/1987

Aprovado para publicação em: 15/9/1987 